\title{
A Software Assistant for User-Centric Calibration of a Wireless Body Sensor
}

\author{
Timm Hörmann, Marc Hesse, Michael Adams and Ulrich Rückert
}

\begin{abstract}
Body sensors have a promising contribution to health promotion in many areas of daily life (telemedicine, corporate health care or recreational sports). However, the valid measurement of vital signs and kinematic data strongly depends on the signals' quality and the users' compliance (proper usage). Although, there is a lot of research work concerning accuracy and calibration of wireless body sensors the human user is typically not involved. Thus, in this work, we present a software assistant (wizard) that guides users during the process of attaching and setting up a wireless body sensor. Furthermore, insights of the implemented software as well as the utilized quality measures and calibration steps are given (ECG, respiration sensor and accelerometer). With the proposed software assistant, the users are instructed to correctly attach the body sensor and calibrate or verify the operability of the various sensor elements. The primary goal is to encourage compliance and the users' sense of control. In this way, we want to reduce faulty operation and ensure optimal signal quality.
\end{abstract}

\section{INTRODUCTION}

Wireless body sensors, e.g. in the form of fitness trackers or smart watches, have a ubiquitous presence in everyday life. From a scientific perspective wireless body sensors are seen as an enabling technology for longitudinal health studies outside of the laboratory. Based on the results it is expected to get a better understanding of human behavior and to encourage a healthy way of life $[1,2]$.

However, the valid measurement of vital signs and reliable recording of kinematic data depends on the calibration and the appropriate usage of the body sensor devices. This involves the correct application of the device as well as the pre-execution of calibration or validation steps. The need to involve the user into the calibration process of a sensor-based ubiquitous computing device has been encourage earlier by [3]. They highlighted the increased users' compliance and improved "sense of control". In this work, we want to promote this idea and present a software assistant that is designed to involve the user into the set-up process.

The intention of making the user responsible for the calibration procedure, is also to prevent operating errors. As a consequence thereof, the risk that an experiment fail or research data has to be discarded is expected to be substantially lower. This idea is based on the conjecture that by explaining the working principles of a device, accidental misuse is avoided. Additionally, the awareness for the operational conditions is presumably increased.

The software assistant proposed in this work is designed to increase the usability of the BG V-4.2 body sensor

Authors are with the Department of Cognitronics and Sensor Systems, CITEC, Bielefeld University, 33619 Bielefeld. E-mail: thoerman@techfak.uni-bielefeld.de

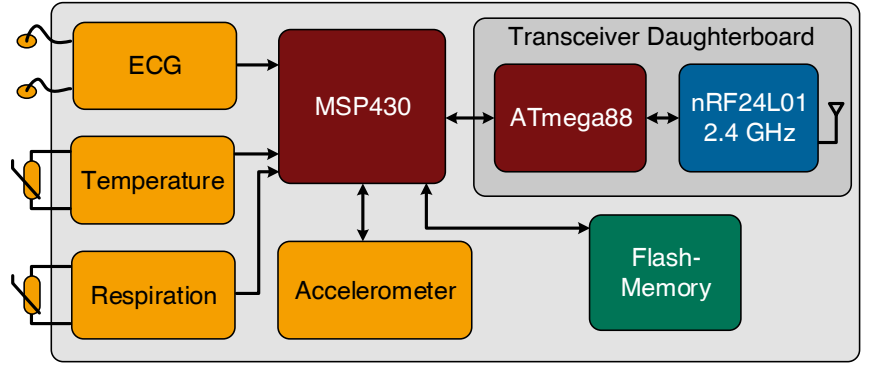

Fig. 1. Technical block diagram of the BG-V4.2 body sensor.

developed at Bielefeld University. Nevertheless, the concept of the assistant could be applied to other devices without constraints.

The BG-V4.2 is a wireless body sensor (WBS, Version: BG-V4.2, fig. 1, which is worn on a chest-strap. It allows to record body movement as well as multiple physiological parameters. In particular we are able to measure a subject's heart rate, respiration rate and upper body acceleration (3axis) $[4,5]$. Additionally, extended parameters such as the estimation of the user's velocity (walking and running) [5] or the physical activity level [6] are calculated.

In this paper, we present a software assistant (wizard) that introduces the WBS's different modes of operation to the user. Furthermore, the users are instructed on how to correctly attach the WBS and tie the provided strap around the chest. The primary goal is to encourage compliance and the sense of control of the users. Thereby, we want to eliminate faulty operation and ensure optimal signal quality.

The paper is structured as follows: First of, in section II. we present the technical design of the WBS along with the recorded parameters. Also, possible disturbances are listed with reference to the included sensors. Furthermore, the need to involve the users into the calibration procedure is argued. Afterwards, in section III the software wizard with the comprised steps of integrity verification and sensor calibration are presented. The expected benefits and goals for further investigations are discussed in section IV A closing summary is part of the discussion and concludes this work.

\section{TECHNICAL BACKGROUND}

In the following, we give insights of the technical aspects of the WBS used in this work sec. II-A. Following up, the parameters of interest, extracted from the raw sensor values, are listed sec. II-B. Additionally, potential causes of malfunction or disturbances are illustrated sec. II-C. 
Finally, we argue the need for a user-centric calibration procedure sec. II-D).

\section{A. Wireless Body Sensor}

The WBS used in this work (BG-V4.2, fig. 1) allows to record a person's heart rate, respiration rate and upper body acceleration. The heart rate is obtained from a 1-lead ECG based on textile electrodes. Respiration rate is extracted from the thoracic movements utilizing a force-sensing resistor [4]. The upper body acceleration is recorded with a MEMS accelerometer (LIS331H, STMicroelectronics), which integrates three sensitive axes. In order to capture accelerations of daily activities as well as sports, its measurement range can be adjusted ( $\pm 6 \mathrm{~g}$ to $\pm 24 \mathrm{~g}$ ).

The whole system is embedded into a single module, which can be used with a conventional chest-strap. The module is powered by a single CR2025 coin cell. All data is stored in a flash memory. It is optionally transmitted through a wireless interface (nRF24L01, Nordic Semiconductor) to a base station. A default sample rate of $100 \mathrm{~Hz}$ is configured for all sensors. A 16-bit microcontroller (MSP430FG4618, Texas Instruments) is utilized to execute the firmware.

\section{B. Parameters}

Based upon the raw sensor readings obtained from the WBS sec. II-A we directly derive various physiological and kinematic parameters:

1) Heart rate and heart rate variability

2) Respiration rate

3) Upper body acceleration and posture

With our WBS heart rate and heart rate variability are calculated via electrocardiography (ECG). This method is based upon the recording of the electrical activity of the heart muscle. We make use of textile electrodes (attached to the chest) that need to be moistened before application.

The respiration rate recording is based on the measurement of thoracic movements. The sensor is based on a forcesensing resistor [4]. The respiration sensor of the BG-V4.2 is directly integrated into a chest-strap.

The upper body acceleration is obtained by an accelerometer, which is integrated into the WBS. In addition to the acceleration, body posture can be calculated from the raw sensor readings (e.g. pitch and roll, see eqn. 1,2).

$$
\begin{aligned}
& \text { pitch }=\operatorname{atan} 2\left(\frac{G_{y}}{\sqrt{G_{x}^{2}+G_{z}^{2}}}\right) \\
& \text { roll }=\operatorname{atan} 2\left(\frac{-G_{x}}{G_{z}}\right) \\
& G_{x}: \text { horizontal axis } \quad G_{y}: \text { lateral axis } \quad G_{z}: \text { vertical axis }
\end{aligned}
$$

\section{Signals and disturbances}

To obtain reliable sensor readings, a variety of disturbances have to be considered. From the functional principle of the sensors, we can determine necessary steps to ensure accuracy and precision. Hereinafter, we give an overview of common disturbances affecting the WBS.
1) Electrocardiogram: Typical disturbances affecting the ECG include electrical interference, electrode contact (and skin conductance) as well as the electrode's positioning [7].

Examples concerning electrical interference are found with the line noise ( $\mathrm{AC}$ noise 50 or $60 \mathrm{~Hz}$ ) or high frequency noise from the electrical activity of the skeletal muscles (EMG noise). To remove such electrical interference analog filters can be used [7]. In our WBS, an analog active low pass filter of 4th order is used (cut-off frequency $33.86 \mathrm{~Hz}$ ). To remove the baseline wander, an additional high pass filter is applied (cut-off frequency $1.56 \mathrm{~Hz}$ ).

Dry electrodes or insufficient skin contact are other common reasons for measurement errors. Regarding chest-strap based textile electrodes proper moistening is required. Additionally, a minimal tension of the chest-strap is needed to ensure good skin contact. Setting the chest-strap's tension is also relevant in terms of motion artifacts, which often occur during vigorous physical activity [8]. Positioning and the electrode contact cannot be corrected by the device itself. Therefore, preceding to an experiment these prerequisites need to be verified by the experimenter or the user.

2) Respiration Sensor: For an optimal signal acquisition of the respiratory curve, the chest-strap must neither be worn too loose nor too tight. Otherwise, the sensor reaches the lower or upper saturation region.

Assuming the respiration sensor is correctly attached to the body, a quasi-periodic waveform can be observed. This waveform follows the breathing movement during inspiration and expiration. However, unlike the activity of the heart muscle, the respiration can be voluntarily controlled by the subject. For instance, the respiration is often interrupted when the subject is speaking. In such a case proper estimation of the respiration rate is impracticable with the proposed method.

3) Accelerometer: The random observational error of the LIS331H accelerometer integrated into the BG-V4.2 is in the order of magnitude of $10^{-3} \mathrm{~g}$. Likewise, systematic observational errors (e.g. caused by temperature fluctuation) are bounded to approximately $10^{-4} \mathrm{~g}$. However, these systematic observational errors are typically dominated by the orientation offset. This offset results from a misalignment between the sensor frame and the body frame that depends on how and where the chest-strap was applied to the user's body $[9,10]$.

\section{Calibration and user perspective}

As shown before (sec. II-C), calibration and validation of WBSs is a mandatory pre-condition in order to guarantee reliability and validity. The impact of noise or other disturbances are widely addressed in recent research. Likewise, methods to detect or minimize the effects of disturbances are proposed:

Concerning heart rate estimation, the effect of noise in the ECG-signal was investigated by [7]. Within their work, they demonstrated the vulnerability of common algorithms used to estimate heart rate, in case the underlying ECG-signal is affected by different types of noise. Furthermore, concerning mobile heart rate acquisition, [8] explain the effect of motion 
artifacts for heart rate variability estimation. In order to detect noisy signals, and thus to prevent false alarms or misleading information, several methods are known to detect the quality of the ECG-signal [11].

Another example for the importance of validation or calibration can be found with the application of accelerometers for physical activity monitoring. For instance [10] examine the impact of the orientation error of an accelerometer used to estimate physical activity. They showed that an orientation error greater than $3^{\circ}$ adversely affects the estimation. Similarly, [12] examine a scenario in which the position of a WBS was interchanged. They demonstrated that without knowing the real position of the WBS, accurate estimation of physical activity was impossible. An overview of different approaches to calibrate accelerometers is given by [13].

Taking these examples, we can argue that without a precedent check-up and calibration of a WBS reliable data is unobtainable. As exemplary pictured above, this applies for accelerometers and ECG recordings. Nevertheless, other sensory elements can be affected as well (e.g. respiration sensor [4]). Following this fact, [3] highlight the importance of "demystifying" the calibration process to the user. They argue the need for clarification, in order to provide a "sense of control" to the users. Thus, prevent insecurities or unnatural behavior. Furthermore, [14] claim that the functionality of a wearable device affects the users' perceived comfort while wearing it. Thus, revealing the functionality of a wearable device to the user is crucial to encourage acceptance (e.g. concerning the way and the location a WBS has to be attached on the body).

Besides the user's compliance and acceptance, other difficulties concerning the usage of WBS for experiments in the field can be identified. These are essential prerequisites like a verification of the state of charge or the documentation of the assignment between subject and device. It is a secondary objective, to emphasize the verification for some of these essential prerequisites to the user as well as to the experimenter.

\section{SOFTwARE AsSistant}

The software assistant (wizard) proposed in this work is used to capture and validate (or calibrate) the electrocardiogram, the respiration signal and the upper body acceleration. It was developed as part of the monitoring software for the BG-V4.2 developed to run on a Windows based computer (http://windows.microsoft.com). The software is written in $\mathrm{C}++$ and makes use of the "Qt" application framework (http://qt.io/).

Using the software, the user is lead through a sequence of steps designed to validate and calibrate the sensors of the BG-V4.2 (fig. 2). In the first step, the WBS is introduced to the user in general. Furthermore, the user is asked to confirm the integrity of the WBS including the chest-strap (sec. III-A). Next, the user is guided through 3 calibration steps (sec. III-B) concerning heart rate, respiration rate and upper body acceleration. The entire procedure takes about 4 minutes if no step has to be repeated.

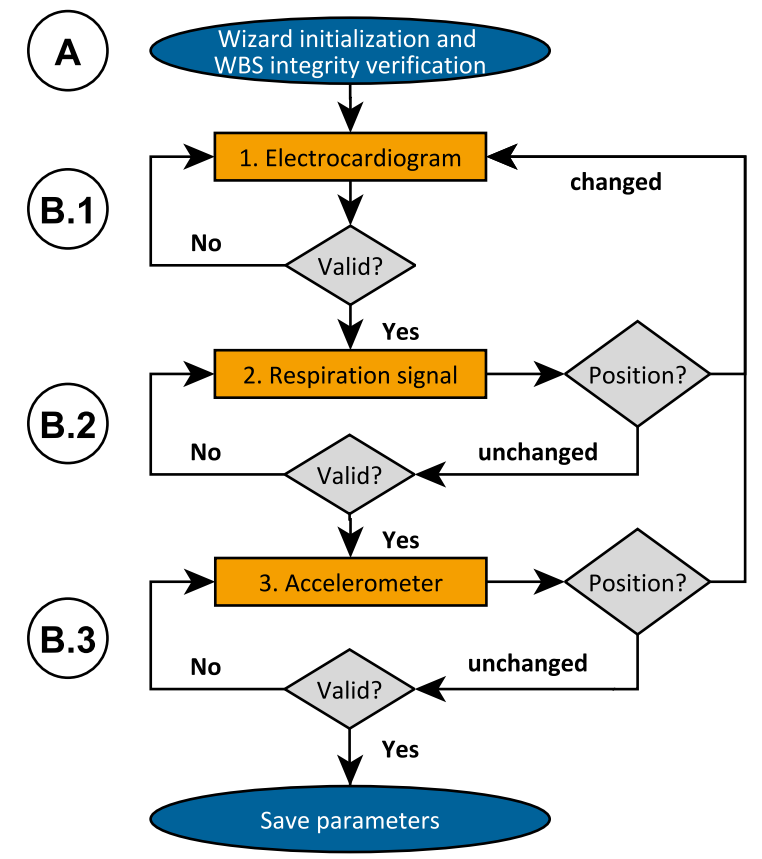

Fig. 2. The wizard's flow diagram. Starting with an initialization phase (A) the following steps are designed to verify the sensor elements (B). If the chest-strap had to be re-positioned, all verification steps have to be repeated.

\section{A. Integrity verification}

During the wizard's initialization, the user is asked to identify the WBS on the basis of its serial number. This step prevents mixing up datasets (assignment between subject and data set). Furthermore, it serves as an initial check-up for the sensors transceiver and the state of charge.

During the integrity verification (fig. 2 A) the user is then asked to keep a check on:

1) Visible damages of the module

2) Visible damages of the chest-strap

3) Identity of displayed and printed serial number

4) State of charge

5) Connection between module and chest-strap

6) Moistening of the textile electrodes

7) Positioning of the chest-strap

Common obstacles in the application of the device are thereby bypassed: Firstly, the assignment between WBS and the subject is documented. Secondly, the fundamental working conditions are guaranteed. This includes that the WBS is switched on and its state of charge has been verified. Furthermore, the module or the chest-strap are inspected in regard to visual damages.

\section{B. Sensor Validation}

1) Heart rate: Various methods have been proposed to assess the quality of the ECG. However, these methods are often developed for clinical assessments and thus are computational demanding. For a WBS with limited resources less complex solutions are preferable. Furthermore, the application scenarios for WBSs are typically only demanding heart rate estimation. Hence, a medical examination is not necessary. 
In order to provide real time information of the ECG quality, we adopted and tuned methods from [15]. In their work, multiple weak metrics are combined to obtain one strong predictor applicable for clinical usage. We partly reused their approach, but only took one of the weak predictors, based on the signals Kurtosis. Yet, Kurtosis is not applicable to detect scattered spikes that often occur due to motion artifacts (chest-strap temporally loses contact to the skin). Therefore, an additional rule was added, which denotes the signal as invalid if the signals range exceeds $75 \%$ of the measuring range (3072 LSB; 12-Bit resolution).

$$
\begin{array}{cl}
\text { Quality }_{\text {ecg }}=\mathrm{K}(\mathrm{ecg}) & \geq 5.4 \wedge \mathrm{R}(\mathrm{ecg}) \leq 3072 \\
\text { Kurtosis: } & \mathrm{K}(X)=\frac{1}{n} \sum_{i=1}^{n}\left(\frac{x_{i}-\bar{x}}{\sigma(X)}\right) \\
\text { Range: } & \mathrm{R}(X)=\max (X)-\min (X)
\end{array}
$$

We validate our quality measure rules eqn. 3 against the MIT-BIH noise stress test database (nstdb) [16]. Each time series in the dataset contains $50 \%$ of noise free and $50 \%$ of noisy data (tbl. I). We found, the quality measure rules to be suitable to detect large disturbances with a signal-tonoise ratio (SNR) lower than $6 \mathrm{~dB}$. Otherwise, for almost undisturbed signals ( $\mathrm{SNR} \geq 18 \mathrm{~dB}$ ) no disturbances are detected. Thus, the signal is marked as valid. Correspondingly, the accuracy and the positive predictive value considerably drop down. However, with a signal-to-noise greater than $6 \mathrm{~dB}$ accuracy is at least $96 \%$. Although, only obvious disturbance are detected with this approach, the number of false alarms (due to false negatives) is limited. The result of the quality metric is presented to the user by adjusting the color of the plotted ECG signal fig. 3. A good signal is colored in red, whereas a disturbed signal is colored in gray.

We conclude that the provided feedback is well suited for non-expert user in terms of identifying most critical

\section{BG V4.2 Assistent - Electrocardiogram}
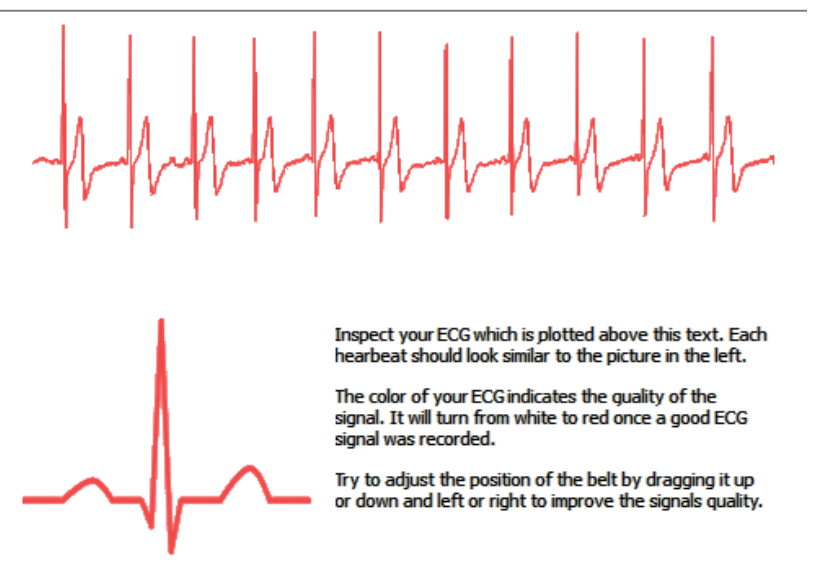

Inspect your ECG which is plotted above this text. Each hearbeat should look similar to the picture in the left.

The color of your ECG indicates the quality of the signal. It will turn from white to red once a good ECG signal was recorded.

Try to adjust the position of the belt by dragging it up or down and left or right to improve the signals quality.
TABLE I

ACCURACCY (ACC), POSITIVE PREDICTIVE VALUE (PPV) AND NEGATIVE PREDICTIVE VALUE (NPV) OF THE ECG QUALITY PREDICTION, EVALUATED AGAINST PHySIONET DATASET [16].

\begin{tabular}{rrrrrrr}
\hline & \multicolumn{3}{c}{ Dataset: nstdb/118 } & \multicolumn{3}{c}{ Dataset: nstdb/119 } \\
SNR & ACC & PPV & NPV & ACC & PPV & NPV \\
\hline No noise & $95 \%$ & $100 \%$ & $95 \%$ & $100 \%$ & $100 \%$ & $100 \%$ \\
$-6 \mathrm{~dB}$ & $96 \%$ & $100 \%$ & $92 \%$ & $100 \%$ & $100 \%$ & $100 \%$ \\
$0 \mathrm{~dB}$ & $96 \%$ & $100 \%$ & $92 \%$ & $100 \%$ & $100 \%$ & $100 \%$ \\
$6 \mathrm{~dB}$ & $96 \%$ & $100 \%$ & $92 \%$ & $97 \%$ & $93 \%$ & $100 \%$ \\
$12 \mathrm{~dB}$ & $93 \%$ & $95 \%$ & $92 \%$ & $64 \%$ & $28 \%$ & $100 \%$ \\
$18 \mathrm{~dB}$ & $76 \%$ & $60 \%$ & $92 \%$ & $50 \%$ & $0 \%$ & $100 \%$ \\
$24 \mathrm{~dB}$ & $51 \%$ & $10 \%$ & $92 \%$ & $50 \%$ & $0 \%$ & $100 \%$ \\
\hline
\end{tabular}

disturbances. These are disturbances that occur if the cheststrap was not applied correctly (e.g. slippery skin contact) or the electrodes need to be moistened.

2) Respiration rate: In order to evaluate the respiration sensor's signal quality, we were able to recourse to a data base from a previously conducted experiment [4]. Thus, for the respiration rate, we have access to reference values obtained from an indirect calorimetry system. Based on this data, we trained a decision tree to estimate the signal's quality by means of deviation to the reference system. We defined the signal quality of our respiration sensor as disturbed, if the deviation between both measurements exceeds $5 \mathrm{~min}^{-1}$ (30 s time window).

With a pruned decision tree, we found the problem to be reduced to one simple decision rule based on the variance. A variance greater or equal to $1137 \mathrm{LSB}$ (approx. $28 \%$ of the amplitude; 12-Bit resolution) was found as a threshold for a valid signal. Invalid respiration signals are discovered with a negative prediction value of $93 \%$ (overall accuracy $72 \%$ ).

\section{BG V4.2 Assistent - Respiration}
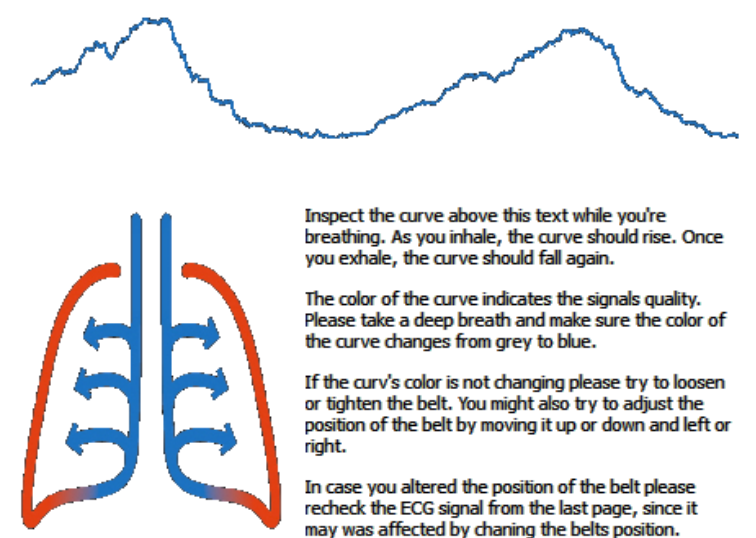

Cancel 2 Back

Fig. 3. ECG validation step of the wizard. Real-time sensory data is displayed (upper corner) along with a reference signal (lower left corner). Once a valid signal was captured the curve in the upper corner changes its color from gray to red.
Fig. 4. Respiration Sensor validation step of the wizard. Real time sensory data is displayed (upper corner). Once a valid signal was captured the curve in the upper corner changes its color from gray to blue. The users is asked to loosen or tighten the chest-strap if the sensor becomes saturated. 


\section{BG V4.2 Assistent - Accelerometer}

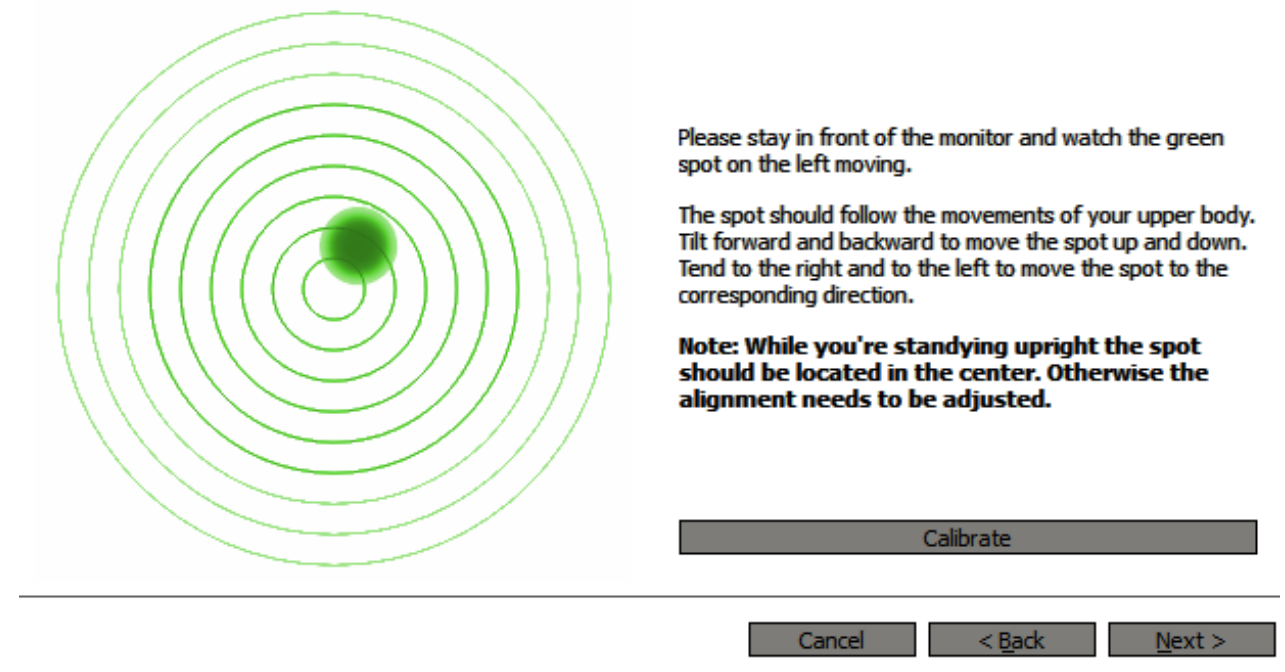

Fig. 5. Accelerometer calibration of the wizard. The user is asked to reposition the sensor until the dot is located in the center of the polar coordinate system. By hitting the calibration button, a calibration procedure is started to gather the correction parameters for the sensor misalignment.

Similar to the ECG quality metric, only obvious deviations are covered. Mostly, this applies if the chest-strap's tension was too slack or taut. Hence, the user is asked to take action only if it is certain that the sensor is not working correctly.

The proposed method can be used to assist the user to find a comfortable yet reliable tautness. In order to further improve the user experience, the signal is denoted as invalid if its mean value is below $25 \%$ of the sensor's measurement range or above $75 \%$, respectively. Similar to the ECG quality, the results of the quality metric for the respiration rate is presented to the user by adjusting the color of the plotted signal (fig. 4). The plotted signal's color changes from gray to blue, if a valid signal was recorded (and vice versa).

3) Acceleration and Body posture: In our applications, we assume the factory calibration of the accelerometer to be sufficiently accurate (sec. II-C). However, the sensor frame and the users body frame need to be aligned. To correct the alignment, we first ask the subjects to manually adjust the sensors position. The subject therefore tracks the alignment by following a dot displayed within a polar coordinate system (fig. 5). The subject's objective is to align the dot as good as possible with the center of the coordinate system.

Next, the acceleration data is recorded while the user is holding a reference position. Therefore, the user is asked to keep its back straight while standing against a wall. The assumption is made that the deviation between the body frame and the sensor frame is high and depends on how the subject applied the sensor. In comparison, we suppose the deviations based on possible variations in the body posture to be comparably small. Apparently, with this approach no clear statement can be made for the "real" alignment. Nevertheless, taking a reference posture to distinguish the users' body frame can improve the inter-individual accuracy. Hence, making it more reasonable to compare datasets among various subjects.
To obtain the rotation matrix between the sensor and the user's body frame, we record up to $10 \mathrm{~s}$ of sensor readings during which the subject is asked to keep the reference posture. If the mean absolute deviation is small $\left(\leq 10^{-6} \mathrm{~g}\right)$, the sensor readings are mapped to a reference vector $v_{r}=[0,0,-1] \mathrm{g}$. Therefore, we make use of methods proposed by [17] based on Rodrigues' rotation formula. An implementation is found in [18] (eqn. 4).

$$
\begin{aligned}
& R(a, b)=I_{3}+v_{x}+v_{x}^{2} \cdot \frac{1-(a \cdot b)}{\left\|v^{2}\right\|} \\
& v=a \times b \quad v_{x}=\left[\begin{array}{ccc}
0 & -v_{3} & v_{2} \\
v_{3} & 0 & -v_{1} \\
-v_{2} & v_{1} & 0
\end{array}\right] \\
& R \quad \text { Rotation matrix } a, b \quad \text { acceleration vectors } \\
& I \quad \text { Identity matrix } \quad v_{x} \quad \text { skew-symmetric cross-product }
\end{aligned}
$$

\section{Discussion}

In this work, we propose the idea of user-centric calibration and validation of a WBS. An software assistant has been developed that guides the users through the checkup and calibration procedure of the BG-V4.2 wireless body sensor. The software combines multiple simplified quality measures (ECG, respiration sensor) and calibrations methods (accelerometer). These measures and calibrations steps are presented to the user in a simplified manner (e.g. signal quality is encoded as color). With the usage of the software assistant we intend to increase the users' compliance.

With the proposed software, we tackle common obstacles that are reported by researches during the conduction of experiments with WBS in field [19]. First off, with the wizard we introduce the WBS to the users. Next, the users are asked to identify the devices (serial number) and to revise the integrity of the device and the chest-strap. Afterwards, the users are guided through three validation and calibration steps. 
Thereby, the users acquaint themselves with the functionality of the device. Furthermore, they are instructed to find an optimal position for the chest-strap and confirm normal operation. Involving the user in the calibration procedure thereby aims to minimize the probability of errors or corrupt data during experiments. Additionally, common operating errors are addressed (e.g. forget to switch the device on or charge it due to inattention). With the assistant it is conceivable that subjects could use the WBS entirely without the help of an expert.

Yet, the provided feedback to the user could be extended. Although, a simplified quality measure limits the risk to overwhelm the user, a more detailed feedback could be beneficial (e.g. concerning the ECG). Instead of just distinguish between valid and invalid signals, a sophisticated quality metric could help the user to discern the concrete type of error (e.g. distinguish whether the ECG is corrupted due to malfunction of the chest-strap or electrical interference). Thus, adaptive support could be provided instead of reporting an undefined error. Regarding body posture, additional hardware, as for instance a gyroscope or magnetometer, could be used to improve the calibration procedure.

The assistant software was already used as part of different experiments. Yet, no separate investigation of its effects on the user or the data integrity was carried out. However, considering first qualitative feedback from our users we expect a positive effect on the users' compliance. For instance, we found the users to be more attentive in handling the device if they had used the assistant software beforehand. Also, we noticed that the users tend to adjust the chest-strap's tautness accordingly to the sensor readings rather than focusing on comfort. Moreover, the software assistant served as a useful tool from the experimenter's perspective. Following the standardized procedure helped to avoid common mistakes. Besides, the assistant was useful for documentation tasks. Furthermore, the defect of a device or single sensor element could be recognized prior to the conduct of an experiment.

For future work, we tend to conduct a quantitative user study. Thereby, we want to evaluate user acceptance and effects on the users' interaction with the WBS in more detail. With the user study remains to be seen, we want to reliably reveal the impacts on the users' compliance and signal quality.

\section{ACKNOWLEDGMENT}

This research was funded by grants from the Cluster of Excellence Cognitive Interaction Technology 'CITEC' (EXC 277), Bielefeld University, the German Federal Ministry of Education and Research (BMBF) within the project "KogniHome" and the Leading-Edge Cluster "Intelligent Technical Systems OstWestfalenLippe" (it's OWL), managed by the Project Management Agency Karlsruhe (PTKA) and the $\mathrm{PhD}$ program "Design of Flexible Work Environments Human-Centric Use of Cyber-Physical Systems in Industry 4.0" supported by the North Rhine-Westphalian funding scheme "Fortschrittskolleg". The authors are responsible for the contents of this publication.

\section{REFERENCES}

[1] G. Appelboom, E. Camacho, M. Abraham, S. Bruce, E. Dumont, B. Zacharia, R. D'Amico, J. Slomian, J. Reginster, O. Bruyere, and E. Connolly, "Smart wearable body sensors for patient self-assessment and monitoring," Archives of Public Health, vol. 72, no. 1, p. 28, 2014.

[2] P. Bonato, "Wearable sensors and systems," Engineering in Medicine and Biology Magazine, IEEE, vol. 29, no. 3, pp. 25-36, May 2010.

[3] R. Johnson, Y. Rogers, N. Bianchi-Berthouze, and J. van der Linden, "Embracing calibration in body sensing: using self-tweaking to enhance ownership and performance," in Pervasive and Ubiquitous Computing (UbiComp 2013), International Joint Conference on. ACM, Sept 2013, pp. 811-820.

[4] M. Hesse, P. Christ, T. Hörmann, and U. Rückert, "A respiration sensor for a chest-strap based wireless body sensor," in IEEE Sensors, Sept 2014, pp. 490-493.

[5] P. Christ and U. Rückert, Identification of Athletes During Walking and Jogging Based on Gait and Electrocardiographic Patterns, ser. Biomedical Engineering Systems and Technologies. Springer, 2014, vol. 452, pp. 240-257.

[6] T. Hörmann, P. Christ, M. Hesse, and U. Rückert, "Robust estimation of physical activity by adaptively fusing multiple parameters," in Wearable and Implantable Body Sensor Networks (BSN), IEEE 12th International Conference on. IEEE, Jun 2015, pp. 1-6.

[7] G. Friesen, T. Jannett, M. Jadallah, S. Yates, S. Quint, and H. Nagle, "A comparison of the noise sensitivity of nine qrs detection algorithms," Biomedical Engineering, IEEE Transactions on, vol. 37, no. 1, pp. 85-98, Jan 1990.

[8] J. Nikolic-Popovic and R. Goubran, "Impact of motion artifacts on heart rate variability measurements and classification performance," in Medical Measurements and Applications (MeMeA), Proceedings of the IEEE International Symposium on, 2013, pp. 156-159.

[9] M. Tundo, E. Lemaire, and N. Baddour, "Correcting smartphone orientation for accelerometer-based analysis," in Medical Measurements and Applications Proceedings (MeMeA), 2013 IEEE International Symposium on, May 2013, pp. 58-62.

[10] L. Wang, S. Su, B. Celler, and E. Ambikairajah, "Analysis of orientation error of triaxial accelerometers on the assessment of energy expenditure," in Engineering in Medicine and Biology Society, 2005. IEEE-EMBS 2005. 27th Annual International Conference of the, Jan 2005, pp. 3514-3517.

[11] B. Aldecoa Sanchez del Rio, T. Lopetegi, and I. Romero, "Assessment of different methods to estimate electrocardiogram signal quality," in Computing in Cardiology, Sept 2011, pp. 609-612.

[12] P. Alinia, R. Saeedi, B. Mortazavi, A. Rokni, and H. Ghasemzadeh, "Impact of sensor misplacement on estimating metabolic equivalent of task with wearables," in Wearable and Implantable Body Sensor Networks (BSN), 2015 IEEE 12th International Conference on, Jun 2015, pp. 1-6.

[13] S.-h. Won and F. Golnaraghi, "A triaxial accelerometer calibration method using a mathematical model," Instrumentation and Measurement, IEEE Transactions on, vol. 59, no. 8, pp. 2144-2153, Aug 2010.

[14] K. Bodine and F. Gemperle, "Effects of functionality on perceived comfort of wearables," in Wearable Computers, 2003. Proceedings. Seventh IEEE International Symposium on, Oct 2003, pp. 57-60.

[15] Q. Li, R. G. Mark, and G. D. Clifford, "Robust heart rate estimation from multiple asynchronous noisy sources using signal quality indices and a kalman filter," Physiological Measurement, vol. 29, no. 1, pp. 15-32, Jan 2008.

[16] A. L. Goldberger, L. A. N. Amaral, L. Glass, J. M. Hausdorff, P. C. Ivanov, R. G. Mark, J. E. Mietus, G. B. Moody, C.-K. Peng, and H. E. Stanley, "PhysioBank, PhysioToolkit, and PhysioNet: Components of a new research resource for complex physiologic signals," Circulation, vol. 101, no. 23, pp. e215-e220, Jun 2000.

[17] J. Hanson, "Rotations in three, four, and five dimensions," ArXiv e-prints, 2011. [Online]. Available: http://arxiv.org/abs/1103.5263

[18] K. Ober, "Calculate rotation matrix to align vector a to vector b in 3d?" Mathematics Stack Exchange, 2014, (version: 2015-08-21). [Online]. Available: http://math.stackexchange.com/q/897677

[19] G.-Z. Yang, J. Andreu-Perez, X. Hu, and S. Thiemjarus, Body Sensor Networks. London: Springer London, 2014, ch. Multi-sensor Fusion, pp. 301-354. 